\title{
Ámbito laboral y bienestar físico en personal de enfermería
}

Marisela Rocío Soria Trujano, ${ }^{1}$ Juana Olvera Méndez, ${ }^{1}$ Edy Ávila Ramos, ${ }^{2}$ Esther María Marisela Ramírez Guerrero, ${ }^{3}$ Enrique Bernabé Cortés Vázquez ${ }^{3}$ y Yasmín Arriaga Abad $^{3}$

\section{Introducción}

La labor que desempeñan los profesionales de enfermería en hospitales implica no sólo sobrecarga de trabajo, sino también el hecho de tener que enfrentarse día a día a situaciones estresantes que pueden afectar su salud y su calidad de vida en general. A pesar de que reciben un entrenamiento específico en el cuidado de enfermos en un ámbito hospitalario, en el que cuentan con apoyo técnico y de otros profesionales, pueden experimentar efectos negativos de este desempeño en su calidad de vida. Realizan su trabajo bajo mucha presión; cada enfermera o enfermero tiene que cuidar a varios pacientes de manera simultánea, lo cual implica someterse a situaciones muy estresantes y esto puede repercutir en su salud. Se han reportado datos que indican que es frecuente encontrar profesionales de la salud que

1 Facultad de Estudios Superiores Iztacala, unam. Psicología Clínica.

2 Facultad de Estudios Superiores Iztacala, unAm. Psicología Métodos Cuantitativos.

3 Facultad de Estudios Superiores Iztacala, UnAm. Psicología Experimental. 
presentan fatiga, somnolencia, baja concentración, dolores de cabeza y musculares, pérdida de peso, etcétera, debido a que el exceso de trabajo los obliga a modificar sus horarios de descanso, de comida y de sueño, así como de distracción y de interacción con familiares y amigos (Cruz et al., 2010; Gil, García y Caro, 2008). El equipo de enfermería, por ejemplo, no tiene descansos acordes con el ciclo biológico y con la vida social, lo cual puede afectar su entorno laboral y extralaboral. Los médicos, enfermeras y asistentes sociales pueden manifestar desgastes físicos y emocionales (Peinado y Garcés, 2004; Enríquez, Sánchez, Santos, Daberkow, Ruiz y Castellanos, 2009). Se ha revelado que si una persona se enfrenta a demandas de trabajo que superen sus recursos para afrontarlas, se verá sometida a una situación de estrés, lo cual puede generarle enfermedades y ausencia laboral, entre otras consecuencias (Mamani, Obando, Uribe y Vivanco, 2007). Rosas (2006) llevó a cabo un estudio y reportó datos que indican que gran parte del equipo de enfermería de un hospital en Hidalgo, México, manifestó estrés, cansancio y falta de realización personal. Escribà y Burguete (2005) mencionan que los principales estresores para el personal de enfermería son: enfrentarse continuamente al sufrimiento de los pacientes, la falta de apoyo por parte de compañeros, la sobrecarga de trabajo, la presión de tiempo, los problemas de interacción con el resto del equipo de enfermería, los conflictos con los médicos y la falta de apoyo en el trabajo. En México, en los hospitales públicos, el número de enfermos atendidos por estos profesionales puede duplicar el número a cargo de los enfermeros(as) de los hospitales privados, y cuentan además con tecnología diferente. Esto indica que el equipo de enfermería desempeña su labor asistencial bajo condiciones de trabajo muy diferentes según el tipo de institución en el que ejerzan su profesión. Así, surgió el interés por realizar el presente estudio cuyo objetivo fue evaluar las relaciones laborales y la presencia de malestares físicos en enfermeras(os) de instituciones de índoles pública y privada. 


\section{Método}

Participantes

Se evaluaron 100 profesionales de enfermería (75 mujeres y 25 hombres); 50 de ellos trabajando en un hospital privado y 50 en instituciones de salud de servicio público (IMSS e ISSSTE), del Distrito Federal o área metropolitana de la Ciudad de México.

Instrumento

Se diseñó una escala tipo Likert de 38 ítems y cinco códigos de respuesta: muy frecuentemente, frecuentemente, pocas veces, casi nunca y nunca. El Alpha de Cronbach fue de .785.

\section{Procedimiento}

Se obtuvo el consentimiento informado de los participantes respetando su anonimato, haciendo de su conocimiento el uso de la información obtenida para presentarla en eventos científicos y/o para publicar. Se evaluó a los participantes de manera individual en su lugar de trabajo.

\section{Resultados}

Los datos se analizaron con la prueba t de Student para muestras independientes con el fin de comparar las variables de interés con cada tipo de institución. La prueba r de Pearson se aplicó para establecer correlación entre el impacto de las relaciones laborales en el bienestar físico. La distribución de normalidad se basó en tres niveles: bajo, moderado y severo; se pretendió medir el impacto del factor institución sobre indicadores de relaciones laborales y de malestares físicos, obteniendo puntuaciones que van de lo mínimo a lo máximo, para poder establecer puntos de corte que den la oportunidad de categorizar a dicho impacto en bajo (menor puntaje alcanzado-menos problemas de rela- 
ción, presencia malestares), moderado, y severo (mayor puntaje alcanzado-más problemas de relación y de malestares físicos).

Los resultados indicaron que $59 \%$ de la muestra general alcanzó un nivel bajo de problemas en las relaciones laborales; $41 \%$ un nivel moderado, y no se reportaron casos con un nivel severo. Cuando se analizaron los datos por institución, no hubo diferencia significativa entre ellas t98 $=-1.490 p>.05$. Considerando los datos porcentuales por institución, se observó que $52 \%$ de los participantes de las públicas alcanzó un nivel bajo en cuanto a problemas en las relaciones laborales; y 48\%, un nivel moderado. Para el caso de la institución privada, 66\% registró un nivel bajo; y 34\%, un nivel moderado. En ambas instituciones no se detectaron casos de nivel severo. En lo que respecta a la presencia de malestares físicos, la muestra general arrojó datos que señalan que $18 \%$ de participantes alcanzaron un nivel bajo de sintomatología; 59\%, un nivel moderado; y 23\%, un nivel severo. Los datos por institución mostraron que en los hospitales públicos, $12 \%$ de participantes obtuvo un nivel bajo; $54 \%$, un nivel moderado; y $34 \%$, un nivel severo; mientras que en la institución privada, $24 \%$ obtuvo un nivel bajo; $64 \%$, un nivel moderado; y $12 \%$, un nivel severo, habiendo diferencia estadística significativa entre ambos lugares de trabajo: t98 $=-2.752 p<.05$, presentando más malestares físicos el equipo de enfermería de las instituciones públicas. Se pudo establecer, además, que en la muestra general, a mayor impacto laboral hubo mayor impacto en el bienestar físico $r=.674 p=.000$. En cuanto al análisis por institución, en el ámbito público se obtuvo una correlación positiva, siendo a mayor impacto laboral, mayor impacto en el bienestar físico $r=.852 p=.000$. De igual forma sucedió en la institución privada $r=.512 p=.000$.

\section{Conclusiones}

A manera de conclusión, se puede decir que en las instituciones públicas se reportaron más problemas en las relaciones laborales y más presencia de malestares físicos. La sobrecarga 
de trabajo en las instituciones públicas y las relaciones laborales conflictivas y/o poco motivantes pueden generar situaciones muy estresantes que tienen efectos sobre la condición física del personal de enfermería. El personal de enfermería tiene que enfrentarse día con día al sufrimiento de los pacientes y, en muchas ocasiones, a la muerte de algunos de éstos, y es evidente que en las instituciones públicas los cuidadores profesionales deben atender a un número superior de enfermos, en comparación con los trabajadores de los hospitales privados. Es importante evaluar incidencia de estrés y ofrecer a este tipo de personal talleres para el afrontamiento a situaciones estresantes, con el fin de optimizar su salud y su rendimiento. Se requiere, además, evaluar destrezas para el manejo de emociones, solución de problemas, asertividad, cambios en los estilos de vida, con el fin de proponer talleres para la adquisición de habilidades que mejoren la calidad de vida del personal de enfermería, puesto que es muy importante que se desempeñe en un nivel alto de ejecución debido a que de estos trabajadores del área de la salud depende el bienestar físico de mucha gente. Los cuidadores profesionales no deben laborar bajo condiciones que impliquen alto nivel de estrés, en un ambiente laboral conflictivo, ni en condiciones de daño físico. Es muy importante que en las instituciones de salud se revisen las condiciones laborales de los empleados de enfermería de manera que se les asignen cargas de trabajo en relación con sus capacidades y con los recursos hospitalarios con los que se cuenten para evitar la vulnerabilidad de este personal a presentar daño en su condición física y, por ende, evitar consecuencias negativas en su desempeño en el manejo y cuidado de enfermos.

\section{Referencias}

Cruz, M.L., Chaves, Y., Barcellos, R., Almeida, L., de Oliveira, I. y Pedräo, L. (2010). Exceso de trabajo y agravios mentales a los trabajadores de la salud. Recuperado de http://scielo. sld.cu/scielo. php?pid=S0864-3192010000100009\&script=sci_arttext. 
Enríquez, C., Sánchez, A., Santos, S., Daberkow, F. Ruiz, M.E. y Castellanos, E. (2009). Estresores laborales en personal de enfermería identificados mediante una escala de adaptación. Desarrollo Cientif Enferm, 17(3), 110-112.

Escribà, V. y Burguete, D. (2005). ¿Cómo perciben las enfermeras de trasplantes las causas de estrés laboral? Index Enfermeras, 14(50), 15-19.

Gil, P., García, J.A. y Caro (2008). Influencia de la sobrecarga laboral y la autoeficacia sobre el Síndrome de Quemarse por el trabajo (burnout) en profesionales de enfermería. Revista Interamericana de Psicología, 42(1), 113-118.

Mamani, A., Obando, R., Uribe, A.M. y Vivanco, M. (2007). Factores que desencadenan el estrés y sus consecuencias en el desempeño laboral en emergencia. Revista Peruana de Obstetricia y Enfermería, 3(1), 50-57.

Peinado, A.I., y Garcés, E.J. (2004). Burnout en cuidadores principales de pacientes con Alzheimer: Síndrome del asistente desasistido. Anales de Psicología, 14(1), 83-93.

Rosas, S. (2006). Síndrome de desgaste profesional (Burnout) en personal de enfermería del Hospital General Pachuca. Revista Científica Electrónica de Psicología, 3(2), 83-105. 